\title{
Lack of significant association between Helicobacter pylori infection and homocysteine levels in patients with cardiac syndrome $X$
}

\author{
Yousef Rasmi ${ }^{1,2}$, Kamal Mehraban ${ }^{3}$, Masoud Sadreddini ${ }^{3}$, Javad Zeynalzadeh ${ }^{4}$, \\ Maryam Majidinia ${ }^{2}$, MirHossein Seyyed-Mohammadzad ${ }^{5}$, Homayoon Babazadeh ${ }^{6}$ \\ ${ }^{1}$ Center for Cellular and Molecular Research, Urmia University of Medical Sciences, Urmia, Iran \\ ${ }^{2}$ Department of Biochemistry, Faculty of Medicine, Urmia University of Medical Sciences, Urmia, Iran \\ ${ }^{3}$ Department of Gastroenterology, Faculty of Medicine, \\ Urmia University of Medical Sciences, Urmia, Iran \\ ${ }^{4}$ Department of Biology, Payame Noor University, P.O. Box 19395-3697, Tehran, Iran \\ ${ }^{5}$ Department of Cardiology, Faculty of Medicine, Urmia University of Medical Sciences, Urmia, Iran \\ ${ }^{6}$ Department of Microbiology, Faculty of Medicine, Urmia University of Medical Sciences, Urmia, Iran
}

\begin{abstract}
Background: Helicobacter pylori (H.pylori) has been implicated in the pathogenesis of several diseases such as cardiac syndrome $X(C S X)$, which includes chest pain, positive exercise stress test and normal angiography. Also, elevation of homocysteine (Hcy) level is associated with CSX, as it can severely disturb vascular endothelial function. We aimed to elucidate whether the infection of H.pylori affect the level of Hcy in CSX.

Methods: Eighty-eight patients with CSX (32 men, 56 women; mean age: $53.8 \pm 11.9)$ and 97 healthy controls (36 men, 61 women; mean age: $45.7 \pm 7.3$ ) were enrolled. Plasma samples were tested for the presence of IgG antibody to H.pylori using enzyme linked immunosorbent assay method. Hcy levels were measured enzymatically.

Results: Plasma Hcy concentration in CSX patients is higher than control group $(13.1 \pm 2.6$ vs. $11.8 \pm 2.5 \mu \mathrm{mol} / \mathrm{L} ; p=0.002$ ). There was no significant difference between Hcy in H.pylori ${ }^{+}$ and H.pylori ${ }^{-}$individuals in CSX group $(13.1 \pm 2.7$ vs. $12.2 \pm 0.6 \mu \mathrm{mol} / \mathrm{L} ; p=0.554)$ and between two groups in controls, respectively $(12.1 \pm 2.2$ vs. $11.4 \pm 2.9 \mu \mathrm{mol} / \mathrm{L} ; p=0.148)$.

Conclusions: Although there is Hcy level increase in H.pylori ${ }^{+}$CSX patients and controls comparing to H.pylori- subjects, but other factors may affect on Hcy level, too. (Cardiol J 2012; 19, 5: 466-469)
\end{abstract}

Key words: cardiac syndrome X, Helicobacter pylori, homocysteine

\section{Introduction}

Cardiac syndrome X (CSX) is a clinical definition, characterized by three main features: angina like chest pain; ST segment depression on treadmill exercise testing and normal coronary arteriography $[1,2]$. In about 10 to $30 \%$ patients who are referred for coronary angiography, does not show vessel stenosis or coronary spasm [3]. The pathogenesis of CSX is still uncertain but microvascular and endothelial dysfunc-

Address for correspondence: Dr. Yousef Rasmi, Center for Cellular and Molecular Research, Urmia University of Medical Sciences, Urmia, Iran and Department of Biochemistry, Faculty of Medicine, Urmia University of Medical Sciences, Urmia, Iran, tel: +984412770698, fax: +984412772360, e-mail: rasmiy@umsu.ac.ir 
tion has been invoked [4]. On the other hand, plasma homocysteine (Hcy) level, which is known to cause endothelial dysfunction and microvascular ischemia were higher in CSX patients [5]. Hcy is a sulphur amino acid formed from methionine during transmethylation, and is either salvaged to methionine by a folate and co-balamin-dependent re-methylation reaction or directed toward degradation by vitamin $\mathrm{B}_{6}$-dependent enzyme cystathionine beta-synthase [6,7]. Also, previous studies has shown an association between viral and bacterial infections including Helicobacter pylori (H.pylori) and some vascular disease such as CSX [8]. H.pylori is a spiral shaped positive gram that cause the most common chronic bacterial infection in the world [9].

Recent data indicate a possible correlation between H.pylori infection and elevated Hcy levels [10]. This study was aimed to examine and reveal the possible relation between $H$.pylori infection and Hcy levels in CSX.

\section{Methods}

\section{Study population}

CSX patients and apparently healthy controls were enrolled. The CSX group consisted of 88 patients (32 men, 56 women). Entry criteria were typical angina chest pain, normal 12-lead ECGs at rest, a positive exercise ECG stress test response and normal coronary angiogram. Non-cardiac causes of chest pain, such as gastrointestinal and musculoskeletal disorders were also investigated and ruled out as appropriate. Patients with diabetes mellitus were not included, since confirmed Hcy increase in diabetes mellitus. The control group consisted of 97 (36 men, 61 women) apparently healthy individuals. None of the controls had a previous history of chest pain or acute/ /chronic diseases. Also, none of controls were taking cardiac or non-cardiac medication. The study was approved by the local research ethics committee and all subjects gave written informed consent.

\section{Study protocol}

A 5-mL tri-sodium-citrated blood sample was obtained from each subject and centrifuged at $2000 \times \mathrm{g}$ for $15 \mathrm{~min}$. Plasma was aliquoted and stored at $-80^{\circ} \mathrm{C}$ until analysis. Anti-H.pylori immunoglobulin- $G$ ( $\operatorname{IgG}$ ) concentration was measured with a commercial enzyme-linked immunosorbent assay (ELISA; Glob anti-HP/IgG, Milan, Italy) according to the manufacturer's instruction (sensitivity $96.5 \%$ and specificity $98.6 \%$ ). The plasma Hcy levels were measured enzymatically (Diazyme, USA). Briefly, oxidized Hcy is first reduced to free
Table 1. The main demographic characteristics of cardiac syndrome X (CSX) and control groups.

\begin{tabular}{lccc}
\hline & CSX & Control & P \\
\hline Sex (male/female) & $32 / 56$ & $36 / 61$ & $>0.05$ \\
Age [years] & $53.8 \pm 11.9$ & $45.7 \pm 7.3$ & 0.001 \\
Body mass index & $27.2 \pm 4.4$ & $26.1 \pm 3.2$ & $>0.05$ \\
{$\left[\mathrm{~kg} / \mathrm{m}^{2}\right]$} & & & \\
\hline
\end{tabular}

Table 2. The prevalence of H.pylori in cardiac syndrome $X(C S X)$ and control groups.

\begin{tabular}{llccc}
\hline \multicolumn{2}{c}{ Age groups [years] } & H.pylori $^{+}$ & H.pylori $^{-}$ & P \\
\hline \multirow{2}{*}{$25-40$} & CSX & $15(100 \%)$ & $0(0 \%)$ & 0.03 \\
& Control & $19(73.1 \%)$ & $7(26.9 \%)$ & \\
$40-55$ & CSX & $31(96.9 \%)$ & $1(3.1 \%)$ & $<0.001$ \\
& Control & $31(50.8 \%)$ & $30(49.2 \%)$ & \\
$>55$ & CSX & $36(87.8 \%)$ & $5(12.2 \%)$ & 0.061 \\
& Control & $6(60 \%)$ & $4(40 \%)$ & \\
\hline
\end{tabular}

Hcy which then reacts with a co-substrate, S-adenosyl methionine (SAM), catalyzed by a Hcy S-methyltransferase. The co-substrate conversion product is amplified by coupled enzymatic cycling reactions. The total Hcy level in the sample is indirectly proportional to the amount of NADH conversion to $\mathrm{NAD}^{+}$.

\section{Statistical analysis}

The data were analyzed by SPSS 16.0 software. Age and BMI were shown as mean \pm standard deviation ( $\mathrm{SD}$ ). The levels of homocysteine were shown as mean \pm standard error of mean (SEM)

The differences between the groups and subgroups were interpreted on the basis of independent-samples $t$-test and for qualitative data on the basis $\chi^{2}$ test. A p-value less than 0.05 was considered statistically significant.

\section{Results}

The main demographic characteristics of the two groups are presented in Table 1 . The mean body mass index was $27.2 \pm 4.4 \mathrm{~kg} / \mathrm{m}^{2}$ in CSX group and $26.1 \pm 3.2 \mathrm{~kg} / \mathrm{m}^{2}$ in control group ( $\left.\mathrm{p}>0.05\right)$.

Eighty two (93.2\%) of CSX patients and 56 (57.7\%) of controls were seropositive for H.pylori $(\mathrm{p}=0.001)$. In this study we divided the patients in 3 groups according to the age including 25-40 years, $40-55$ years and over 55 years (Table 2 ). In 25-40 years and 40-50 years group there were 
Table 3. Plasma homocysteine level in cardiac syndrome $\mathrm{X}(\mathrm{CSX})$ and control groups.

\begin{tabular}{lllc}
\hline & H. pylori & H. pylori & P \\
\hline Control $[\mu \mathrm{mol} / \mathrm{L}]$ & $12.1 \pm 2.2$ & $11.4 \pm 2.8$ & 0.148 \\
CSX $[\mu \mathrm{mol} / \mathrm{L}]$ & $13.1 \pm 2.7$ & $12.2 \pm 0.6$ & 0.554 \\
\hline
\end{tabular}

a significant difference between prevalence of antiH.pylori positive (H.pylori ${ }^{+}$) and anti-H.pylori negative (H.pylor $\left.i^{-}\right)$among CSX and controls ( $\mathrm{p}=0.03$ and $\mathrm{p}<0.001$, respectively). But there was no significant difference in $>55$ group $(\mathrm{p}=0.061)$.

In CSX and control groups the mean level of Hcy was $13.1 \pm 2.6$ and $11.8 \pm 2.5 \mu \mathrm{mol} / \mathrm{L}$, respectively $(\mathrm{p}=0.002)$. On the other hand, the mean Hcy levels in H.pylori ${ }^{+}$and H.pylori ${ }^{-}$individuals of CSX group was $13.1 \pm 2.7$ and $12.2 \pm 0.6 \mu \mathrm{mol} / \mathrm{L}$, respectively $(\mathrm{p}=0.554)$. In control group, the mean Hcy levels of $H . p y l o r i^{+}$and $H . p y l o r$ individuals, was $12.1 \pm 2.2$ and $11.4 \pm 2.9 \mu \mathrm{mol} / \mathrm{L}$, respectively $(\mathrm{p}=0.148$; Table 3$)$. Hcy level in H.pylori ${ }^{+}$individuals tend to increase but it is not significant in comparison with $H$.pylori individuals.

\section{Discussion}

Many studies showed that the elevated Hcy is associated with endothelial dysfunction and contributes to increased risk of cardiovascular diseases [11]. These effects are mediated by its cytotoxic effects on endothelial cells, stimulation of platelet adhesion and/or its promotion of pro-coagulant activity [12]. There is some evidence that thiols react in the presence of NO to form S-nitrosothiols, compounds with vasodilatory and antiplatelet effects [13]. Timurkaynak et al. [5] reported that plasma Hcy level, which is known to cause endothelial dysfunction and microvascular ischemia were higher in CSX patients.

In addition, Hcy metabolism involves a complex interaction between folate and vitamin $B_{12}$. So chronic H.pylori infection causes decreased absorption of both folate and vitamin $\mathrm{B}_{12}$ and this condition is associated with hyperhomocysteinemia [7]. In this study because of some limitation we could not measure folate and $\mathrm{B}_{12}$ levels in plasma.

Multiple pathophysiologic abnormalities have been reported in patients with CSX. The most convincing evidence includes generalized endothelial dysfunction and inflammation [14]. In addition
H.pylori recently has been associated with CSX [15]. Mendall et al. in 1994, for the first time showed that H.pylori seropositivity was twice as common in coronary arteries disease patients as in control subjects [9]. Since then, many studies have been demonstrated an association between H.pylori infections and some vascular diseases, such as ischemic heart disease [16]. Chronic infection may be accompanied by persistently increased production of inflammatory metabolites [8]. H.pylori produces some antigenic substances, including heat shock protein, urease, and lipopolysaccharide, all of which can be taken up and processed by lamina properia macrophages and active $T$-cells and cause increased production of inflammatory cytokines such as interleukine-1 (IL-1), IL-6, tumor necrosis factor alpha $(\mathrm{TNF} \alpha)$, and most important IL-8. These agents may affect vessel motility and induce endothelial dysfunction and microvascular hyperconstriction [15]. On the other hand, Elizade et al. in 1997 showed that H.pylori infection induces an increase in the flux of leukocytes and in the appearance of platelet and leukocyte-platelet aggregates in gastric venules in an animal model, so platelet activation and aggregation contribute to the associated microvascular dysfunction and inflammatory cell recruitment [17].

The main finding of the present study is that patients with CSX showed increased Hcy levels as compared to normal controls. The frequency of $H . p y l o r i$ infection was also increased in patients with CSX, and the presence of H.pylori infection did not affect the Hcy level significantly.

Because there is no consistent evidence, the hypothesis that H.pylori causes increased Hcy level in patients with CSX was still controversial. Recent studies reported no significant difference in blood Hcy level in H.pylori ${ }^{+}$and H.pylori ${ }^{-}$individuals [18]. Our analysis showed the similar results indicating that there is no link from H.pylori infection to increased Hcy levels in CSX patients. We suggest that the postulated link between $H$.pylori and CSX [19], if it is actually exists, is unlikely to be mediated through elevated Hcy levels.

\section{Conclusions}

Although there is an increase in Hcy level in H.pylori ${ }^{+}$in CSX and controls comparing to H.pylori ${ }^{-}$ subjects, but other factors may affect on Hcy level.

Conflict of interest: none declared 


\section{References}

1. Hurst T, Olson TH, Olson LE, Appleton CP. Cardiac syndrome X and endothelial dysfunction: New concepts in prognosis and treatment. Am J Med, 2006; 119: 560-566.

2. Slobodny PR. Cardiac syndrome X. Dimens Crit Care Nurs, 2008; 27: 209-212.

3. Li AL, Lee BC, Chen KC, Weng CS, Chu SH. Brachial artery flow-mediated vasodilation in patients with cardiac syndrome X. Angiology, 2008; 59: 581.

4. Bellamy MF, Goodfellow J, Tweddel AC, Dunstan FD, Lewisb MJ, Henderson AH. Syndrome X and endothelial dysfunction. Cardiovasc Res, 1998; 40: 410-417.

5. Timurkaynak T, Balcioglu S, Arslan U, Kocaman SA, Cengel A. Plasma homocysteine level in cardiac syndrome $\mathrm{X}$ and its relation with duke treadmill score. Saudi Med J, 2008; 29: 364-367.

6. Leung WK, Ma PK, Choi PCL et al. Correlation between Helicobacter pylori infection, gastric inflammation and serum homocysteine concentration. Blackwell Science Ltd, 2001; 6: 146-150.

7. Nasri H. Close associaton between helicobacter pylori infection and serum homocysteine in stable hemodialysis patients. Adv Mol Med, 2005; 1: 171-175.

8. Rasmi Y, Raeisi S, Seyyed Mohammadzad MH. Association of inflammation and cytotoxin-associated gene A positive strains of Helicobacter pylori in cardiac syndrome X. Helicobacter, 2011; 17: 116-120.

9. Mendall MA, Goggin PP, Molineaux N et al. Relation of Helicobacter pylori infection and coronary heart disease. Br Heart J, 1994; 7: 437-439.

10. Ozer B, Serin E, Gumurdulu Y et al. Helicobacter pylori eradication lowers serum homocysteine level in patients without gastric atrophy. World J Gastroenterol, 2005; V11: N18.

11. Evrengul H, Tanriverdi H, Kuru O et al. Elevated homocysteine levels in patients with slow coronary flow: relationship with Helicobacter pylori infection. J Compilation, 2007; 12: 298-305.

12. Soysal D, Savafl S, Susam B et al. The association of plasma homocysteine, cardiac risk factors and serum nitrite in patients with coronary artery disease, cardiac syndrome $\mathrm{X}$ and healthy subjects. Anadolu Kardiyol Derg, 2003; 3: 26-34.

13. Stamler JS, Osborne JA, Joraki O et al. Adverse vascular effects of homocysteine are modulated by endothelium derived relaxing factor and related oxides of nitrogens. J Clin Invest, 1993; 91: 308-318.

14. Hurst T, Olson T, Olson L, Appleton C. Cardiac syndrome X and endothelial dysfunction: New concepts in prognosis and treatment. Am J Med, 2006; 119: 560-566.

15. Eskandarian R, Malek M, Mousavi S H, Babaei M. Association of Helicobacter pylori infection with cardiac syndrome X. Singapore Med J, 2006; 47: 705.

16. Nocente R, Gentiloni N, Cremonini F et al. Resolution of syndrome $\mathrm{X}$ after eradication of virulent cag A-positive helicobacter pylori. Southern Med J, 2000; 93: 10.

17. Elizalde JI, Gomez J, Panes J et al. Platelet activation in mice and human Helicobacter pylori infection. J Clin Invest, 1997; 100: 996-1005.

18. Itou S, Goto Y, Kondo T, Nishio K, Kawai S, Ishida Y. No associations of Helicobacter pylori infection and gastric atrophy with plasma total homocysteine in Japanese. Internat J Med Scien, 2007; 4: 2.

19. Rasmi Y, Raeisi S. Possible role Helicobacter pylori infection via microvascular dysfunction in cardiac syndrome X. Cardiol J, 2009; 16: 585-587. 